\title{
NousRire : étude de cas d'une entreprise québécoise repensant la marque responsable traditionnelle
}

\author{
Audrey Girard ${ }^{a}$, Jonathan Deschênes ${ }^{b}$
}

RÉSUMÉ. Fondée en 2015, NousRire est une petite entreprise à but lucratif québécoise qui a pour mission de « rendre accessibles des aliments biologiques non périssables d'excellente qualité, tout en permettant de faire des économies et en créant un impact positif sur la Terre et sur ses habitants $»^{1}$. L'entreprise offre aux clients de passer leurs commandes en ligne et de venir les récupérer dans des lieux de cueillette précis durant des périodes appelées « journées d'emballage ». Ces aliments sont disponibles en vrac pour minimiser la production de déchets. Plus de 500 bénévoles œuvrent durant ces périodes afin de distribuer la nourriture dans les contenants des clients, qui emballent eux-mêmes leur commande. NousRire est constituée de 18 « cellules » autogérées ancrées dans diverses régions du Québec, dont l'Estrie, l'Abitibi, les Laurentides et la région de Montréal. L'entreprise fonctionne grâce à l'implication de 2000 bénévoles et d'environ 20 employés.

\begin{abstract}
Founded in 2015, NousRire is a small, profit-making Quebec company whose mission is to "make accessible excellent quality non-perishable organic food, while saving money and creating a positive impact on the Earth and its inhabitants". The company gives customers the opportunity to order online and pick up their items at specific locations on "packing days." The food is available in bulk to minimize waste generation. Over 500 volunteers work on these days to distribute food in the containers of customers who pack their orders themselves. NousRire consists of 18 self-managed "cells" established in various regions of Quebec, including Estrie, Abitibi, the Laurentians and Montreal. The business operates with the participation of 2000 volunteers and approximately 20 employees.
\end{abstract}

\section{Introduction}

NousRire ${ }^{2}$ est une entreprise s'inscrivant dans la démarche des marques responsables ${ }^{3}$. Il n'existe pas une définition unique de la marque responsable, mais elle implique en général d'intégrer la notion de responsabilité sociale à la promesse de la marque (Stewart, 2011). Dans un contexte où la concurrence entre les marques est plus féroce que jamais et où les facteurs de différenciation sont de plus en plus ténus, la dimension de responsabilité sociale de la marque prend aussi un sens supplémentaire en permettant à la marque de générer un élément de différenciation aux yeux des consommateurs (Pringle et Thompson, 2001). De plus en plus d'entreprises se tournent donc vers la responsabilité sociale afin de repositionner leur marque grâce à une modification de leurs activités qui leur permet de diminuer l'empreinte écologique ou de devenir de meilleurs citoyens corporatifs. De nombreux ouvrages présentent des outils pour soutenir les marques dans cette aventure (Fuller, 1999; Belz et Peattie, 2012; Robertson, 2014).

La littérature s'intéressant aux marques responsables a principalement démontré que les actions spécifiques de ces marques sont généralement tournées vers l'environnement externe à l'entreprise, c'est-à-dire qu'elles se concentrent sur des activités précises, comme la réduction de la pollution, l'aide aux populations défavorisées, la santé, etc. (Kotler et Lee, 2005). On n'a qu'à penser à l'entreprise Toms, qui remet gratuitement une paire de souliers pour chaque soulier vendu ou encore à Starbucks et son programme d'achat de café équitable.

\footnotetext{
${ }^{a}$ Doctorante en sciences humaines appliquées, Université de Montréal

b Professeur agrégé en marketing, département de marketing, HEC Montréal
} 
Or, toutes les marques ne conçoivent pas la responsabilité uniquement par des actions spécifiques tournées vers l'environnement externe. Certaines créent leur valeur de responsabilité de manière plus holistique afin de maximiser le rayonnement de cette valeur dans toutes les facettes de la marque.

L'objectif de cet article est ainsi d'explorer comment des marques peuvent développer une proposition de valeur responsable globale. Plus spécifiquement, grâce à l'étude du cas de NousRire, cet article répond à la question suivante : Comment une marque peut-elle pleinement déployer son potentiel de responsabilité afin d'apporter des bénéfices autant à l'interne qu'à l'environnement externe dans lequel elle évolue? Nous verrons aussi que ce déploiement du potentiel de responsabilité amène la marque à remettre en question la vision traditionnelle de l'économie marchande (laquelle sera décrite dans la prochaine section).

Le reste de l'article se développe comme suit : tout d'abord, nous présentons la logique traditionnelle de la marque responsable dans la littérature en marketing, qui suggère que ce type de marque concentre ses actions vers l'environnement externe et implique une vision classique de l'économie marchande. Par la suite, nous présentons la méthode utilisée pour développer l'étude de cas. Nous élaborons ensuite sur les fruits de notre analyse et démontrons que NousRire, par ses actions variées, transcende l'approche traditionnelle de la marque responsable en développant une proposition de valeur créative basée sur le " potentiel humain » qui implique l'adoption de pratiques créatives au travail, la modification de la conception du client et la transformation de sa gouvernance. Finalement, nous proposons une réflexion globale sur ce type de marque et sur les dérives potentielles pouvant être associées à ses pratiques.

\section{Logique traditionnelle de la marque responsable}

Divers auteurs en marketing se sont intéressés à conceptualiser la marque responsable. L'analyse de leurs écrits permet de conclure que ce type de marque comporte des attributs récurrents qui se résument sous deux constats globaux.

Comme premier constat, la marque responsable est généralement tournée vers l'environnement externe à l'entreprise. Ce constat se manifeste de trois façons.
Tout d'abord, la marque responsable offre normalement une proposition centrée sur un respect de l'environnement grâce, notamment, à l'emploi d'ingrédients plus écologiques et de produits recyclés, réusinés, biodégradables, etc. afin de réduire son empreinte écologique (Fuller, 1999; Iannuzzi, 2012; Murray, Skene et Haynes, 2017). De surcroît, la marque responsable offre aussi fréquemment des solutions spécifiques à des problématiques sociétales comme la santé, l'éducation, la faim, la pauvreté, la maladie, etc. Elle met alors en place certaines stratégies, comme la promotion de messages prosociaux, la vente de produits soutenant des causes et la philanthropie corporative (Kotler et Lee, 2005; Austin et Seitanidi, 2014). Finalement, elle se concentre aussi parfois sur la création de produits de plus grande valeur pour les consommateurs, notamment grâce à des composantes sécuritaires et durables, à une simplicité de compréhension et d'usage ainsi qu'à la possibilité d'intégrer les produits à l'écosystème existant des produits possédés par le consommateur (Samli, 1992).

Comme second constat, la marque responsable s'ancre dans une vision classique de l'économie marchande. En effet, dans cette logique, la marque a pour fonction principale la production et/ou la mise en marché d'un produit ou service (associé à une proposition de valeur responsable) qui sera ensuite acquis et consommé. Le consommateur est ainsi conceptualisé comme « être consommant » responsable d'acheter, de consommer, et, finalement, de se débarrasser du produit. Dans l'économie marchande classique, les rôles sont ainsi clairs (Ertz, Hallegatte et Bousquet, 2019). Cette logique traditionnelle de l'économie marchande valorise grandement la dimension économique des échanges.

En effet, la marque responsable doit développer une proposition de valeur concurrentielle forte sur un marché multimarque complexe et la dimension de la responsabilité peut - et doit - devenir un atout commercial pour la marque. La littérature insiste d'ailleurs sur l'importance d'une «responsabilité intéressée ». Selon ce principe, les marques ne deviennent pas responsables par devoir ni par obligation morale, mais plutôt pour des raisons stratégiques (Kotler et Lee, 2005). Cela implique que la rentabilité financière de la marque doit primer sur les motivations purement altruistes (Fuller, 1999; Samli, 1992). L'objectif est donc de 
produire le plus de biens possible socialement, mais aussi et surtout économiquement : augmenter les ventes et les parts de marché; attirer, motiver et retenir les employés; augmenter l'attrait de l'entreprise pour les investisseurs; diminuer les coûts de transaction; augmenter l'innovation; créer des incitatifs au réseautage; etc. (Kotler et Lee, 2005; Champniss et Vilà, 2011).

De plus, la responsabilité doit être intégrée à une stratégie d'ensemble visant à renforcer le positionnement et la différenciation de la marque sur le marché (Pringle et Thompson, 2001; Porter et Kramer, 2006). Dans un environnement hautement compétitif où il est difficile pour une marque de se faire voir par les consommateurs, la responsabilité devient un marqueur potentiel de différenciation. Ces initiatives responsables s'inscrivent donc dans l'idéologie marchande du « gagnant-gagnant » : se faire $d u$ bien à soi, comme organisation, en faisant le bien.

L'objectif de cette étude est donc de démontrer que certaines marques déploient des pratiques allant au-delà de cette logique traditionnelle de la marque responsable. Grâce à l'étude du cas de NousRire, nous verrons comment une entreprise peut déployer plus globalement sa valeur de marque afin de rayonner, autant à l'interne qu'à l'externe, et de remettre en question la vision classique de l'économie marchande.

\section{Méthodologie}

L'étude de cas est une méthode de recherche qualitative utilisée en sciences sociales qui offre la possibilité de produire une analyse détaillée et approfondie de cas spécifiques (Eisenhardt, 1989). Ce genre d'étude contient typiquement une combinaison de données (p. ex., archives, entrevues, observations et questionnaires) et est utilisé afin d'accomplir différents buts, comme fournir une description détaillée ou encore tester ou générer une théorie (Eisenhardt, 1989). L'étude de cas peut être constituée d'un cas unique d'importance ou être déployée grâce à un design impliquant l'analyse de plusieurs cas (Yin, 2017). La présente étude a pour objectif de fournir une description détaillée de NousRire dans le but de mettre l'accent sur le caractère singulier de cette entreprise.

Dans cette étude, nous avons utilisé une combinaison de diverses méthodes. Premièrement, une analyse des documents internes concernant l'identité et les rôles des acteurs ainsi que les politiques organisationnelles a été effectuée. Nous avons intégré ces données à notre analyse afin de bien comprendre la constitution de l'organisation et son fonctionnement.

Par la suite, nous avons procédé à des séances d'observation dans le but de mieux comprendre la dynamique organisationnelle et les pratiques de la marque. Pour ce faire, nous avons réalisé deux séances d'observation de deux heures chacune: une en période de "préemballage ${ }^{4}$ et l'autre durant une «journée d'emballage». Au total, les actions et interactions d'environ 15 employés, 20 clients et 10 bénévoles ont été observées.

Finalement, trois entretiens semi-dirigés ont été réalisés avec des gestionnaires ayant des responsabilités significatives dans l'organisation ${ }^{5}$. Nous avons aussi réalisé deux courts entretiens avec des bénévoles lors des observations.

La collecte de données a été réalisée entre août et septembre $2019^{6}$. Les données ont été analysées de manière inductive afin de permettre une découverte progressive des pratiques liées à la marque (Strauss et Corbin, 1998). L'identification des pratiques responsables a été développée en contrastant les différentes sources de données collectées (Schrier, 2012). Pour ce faire, nous avons noté les répétitions, similitudes et différences dans les données (Bryman, 2015). Les pratiques ainsi analysées ont été progressivement codées et regroupées sous les trois grandes catégories décrites dans la section suivante.

\section{Analyse des pratiques de NousRire}

Au premier regard, il est possible de constater que NousRire se positionne comme une marque offrant une valeur tournée vers l'environnement et la santé des consommateurs, grâce à une offre de produits biologiques de qualité et à un format en vrac permettant de limiter la production de déchets associés au suremballage des produits (comme présenté en introduction). Or, une analyse plus approfondie révèle que l'entreprise ne s'est pas restreinte à ce positionnement. Elle a en fait choisi de développer une proposition de valeur axée sur la notion de "potentiel humain», qui implique l'épanouissement des acteurs, leur imputabilité, le sens des initiatives et la qualité des relations humaines. Comme l'exprime un des gestionnaires de l'entreprise : 
Les personnes sont encouragées à s'épanouir au sein de l'organisation, à développer leur plein potentiel en prenant des responsabilités et des initiatives, en améliorant leur capacité à entrer en relation avec les autres, en étant amenées à se connaître mieux, en apprenant à bien verbaliser leurs tensions. (Gestionnaire 1)
Cette notion de potentiel humain a de vastes ramifications au sein de la marque et implique son développement selon trois axes fondamentaux résumés dans le Tableau 1 . Nous présenterons de manière détaillée les pratiques associées à chacun de ces axes.

\begin{tabular}{|c|c|}
\hline $\begin{array}{l}\text { Axe du déploiement de } \\
\text { la marque responsable }\end{array}$ & Pratiques dans l'organisation \\
\hline $\begin{array}{l}\text { Adoption de pratiques } \\
\text { créatives en milieu de } \\
\text { travail }\end{array}$ & $\begin{array}{l}\text { Pratiques de socialisation : } \\
\text { - Intégration du jeu aux activités quotidiennes } \\
\text { - Création du comité Culture et plaisir } \\
\text { - Routine du Cercle du matin } \\
\text { Pratiques d'attention : } \\
\text { - Technique pour faire bouger les employés } \\
\text { - Pratique de l'observateur } \\
\text { - Pauses ritualisées } \\
\text { - Vocabulaire réfléchi }\end{array}$ \\
\hline $\begin{array}{l}\text { Modification de la } \\
\text { conception du client }\end{array}$ & $\begin{array}{l}\text { - Client apportant ses propres contenants et responsable du transport de } \\
\text { quantités significatives de denrées } \\
\text { - Client adoptant le rôle de bénévole pour l'organisation en échange d'un rabais } \\
\text { sur les achats futurs } \\
\text { - Partage des données financières de l'entreprise avec les bénévoles/clients }\end{array}$ \\
\hline $\begin{array}{c}\text { Transformation de la } \\
\text { gouvernance et des rôles } \\
\text { clés de gestion }\end{array}$ & $\begin{array}{l}\text { - Adoption du modèle de gouvernance holacratique } \\
\text { - Constitution collective du Guide du vivre ensemble } \\
\text { - Développement d'un poste dédié au potentiel humain } \\
\text { - Développement d'un poste dédié au rayonnement }\end{array}$ \\
\hline
\end{tabular}

Tableau 1 - Pratiques de potentiel humain de la marque NousRire

\subsection{Adoption de pratiques créatives en milieu de travail}

Tout d'abord, au fil des ans, la marque a développé diverses pratiques créatives axées sur la socialisation et l'attention. Premièrement, certaines pratiques ciblent la socialisation des employés, notamment en valorisant le jeu et des pratiques ludiques durant la journée de travail. En effet, les travailleurs jouent parfois à des jeux de société, à des jeux de cartes, etc. sur l'heure du midi. En réunion, il n'est pas rare non plus de «jouer» à se passer le chapeau pour animer les échanges. De manière plus formelle, un comité Culture et plaisir a été créé pour organiser des événements ludiques entre travailleurs. Ce comité a par exemple conçu les Olympiades NousRire, qui impliquent différentes épreuves, dont certaines sont décrites par un des gestionnaires :
On a organisé les Olympiades NousRire par le comité Culture et plaisir. On avait le lancer du pois chiche dans un petit bocal. On a eu une espèce de course sur les petits chariots qu'on a pour transporter les choses. Il y avait une personne sur le chariot avec plein de boittes et il fallait contourner les bidons. C'était comme une course à relais. [...] C'était au mois de juin et c'était vraiment le fun. L'idée, c'était de sortir de la routine quotidienne, de créer plus de liens. (Gestionnaire 1)

De plus, les travailleurs sont aussi accueillis dans un "Cercle du matin » permettant de commencer la journée ensemble en déjeunant et en ayant des discussions communes. Un tour de table d'environ 15 minutes concernant une question préalablement établie est une pratique courante du Cercle du matin. Par exemple, les employés peuvent être 
amenés à «décrire une nouvelle planète » de manière imaginaire, simplement pour générer une conversation ludique, inclusive et créative.

Dans un deuxième temps, certaines pratiques sont utilisées pour favoriser l'attention. D'abord, les employés sont incités à bouger durant les réunions pour optimiser l'écoute et l'attention. Ils sont encouragés à se lever et même à sauter sur place plusieurs fois pendant les réunions. De plus, toujours en période de réunion, les employés utilisent parfois la pratique de l'observateur pour prendre du recul sur les discussions. Cette pratique est initiée par l'agitation d'une cloche. Elle est décrite par un gestionnaire de la manière suivante :

Quand on est en train de stagner dans la discussion ou qu'il commence à y avoir des tensions interpersonnelles qu'on ne comprend pas trop, eh bien là, on sonne la cloche. On recule, parfois on change de place pour observer d'une perspective différente. (Gestionnaire 3)

Les travailleurs, les bénévoles et les clients sont aussi invités à prendre diverses pauses ritualisées encourageant « un retour dans le corps » menant à une plus grande présence attentionnée. Parmi elles, la pause Respire et la pause Mangoustan sont les pratiques les plus ancrées dans l'organisation. La pause Mangoustan est une pause ludique qui invite tous les participants à cesser leurs activités et à danser ensemble le temps d'une chanson. La pause Respire invite, au son d'une cloche, tous les participants à cesser leurs activités et à diriger leur attention vers leur respiration pendant une minute. Ces pauses, en invitant un "passage du cérébral au corps ", favorisent un esprit clair et attentif ainsi qu'une ouverture sur les autres. La pause Respire, en incitant à se concentrer sur la respiration, est une pratique qui favorise la pleine conscience, c'est-àdire l'état d'esprit qui émerge lors d'une attention volontaire et continue portée sur le moment présent sans jugement (Kabat-Zinn, 2013). Cette qualité de la conscience amène plusieurs bienfaits, dont une augmentation de la concentration, une réduction du stress et de l'anxiété ainsi qu'une augmentation de la compassion envers soi et les autres (Davis et Hayes, 2011).

Une cliente bénévole raconte son expérience de ces pauses :
C'est important de déconnecter un peu et de prendre une distance, de rentrer en toi. Juste une pause Respire et après tu te sens vraiment mieux pour reprendre. Tu te rappelles qu'il ne faut pas que tu te mettes autant de pression. Au début, tu veux tellement être bon et efficace. Ici, j’ai appris à prendre mon temps, à plus être dans le moment et à plus parler avec les gens qui viennent chercher leur commande. Je suis moins stressée. Je prends mon temps et advienne que pourra. (Bénévole 1)

Cette personne ajoute ensuite que ces pauses ont même une répercussion dans d'autres sphères de sa vie: "J'ai vu aussi un modèle d'entreprise que j'aimais beaucoup et j'essayais même de reprendre des pratiques d'ici, comme la pause Respire, et de ramener ça dans mon travail à moi. »

Finalement, l'organisation s'est dotée d'un « vocabulaire réfléchi » ayant pour objectif l'emploi conscient d'une terminologie s'éloignant délibérément des termes organisationnels classiques et visant à connecter profondément le langage quotidien aux valeurs de l'organisation. Ce vocabulaire est reflété dans les écrits ainsi que dans l'oralité. Nommé «Lexique typiquement NousRire », il est disponible sur le Web et permet aux acteurs de se familiariser avec certains termes centraux pour l'entreprise ${ }^{7}$. Par exemple, le terme « œuvrer » est utilisé au lieu de «travailler» et « rieurs/rieuses » désigne toutes les personnes qui s'impliquent chez NousRire. Un gestionnaire explique l'importance de ce vocabulaire : «On est intègre par rapport à ce qu'on vit et on veut être intègre dans notre manière de communiquer. On veut que les mots fittent avec qui on est. » (Gestionnaire 3)

Globalement, la plupart de ces pratiques ont émergé naturellement, c'est-à-dire qu'il n'y a pas eu d'efforts déployés spécifiquement dans le but de les mettre en place. Ce sont souvent des pratiques qui faisaient partie de la vie personnelle des gestionnaires et qui se sont transposées à l'organisation. Parfois, ce sont des membres d'équipe qui les ont initialement amenées, comme les jeux sur l'heure du midi. Une chose est certaine, pour que la pratique s'ancre, il faut qu'elle soit organiquement adoptée par le groupe, comme l'indique un gestionnaire : "On a des pratiques qu'on a semées au début et certaines ont grandi, et d'autres non. Ce 
sont des pratiques qu'on fait naturellement dans notre vie. » (Gestionnaire 3)

\subsection{Reconcevoir le concept de client}

Dans un commerce traditionnel, le consommateur se présente en magasin, fait l'achat de ses biens préemballés et les rapporte à son domicile, souvent dans un emballage supplémentaire. NousRire, de par la dimension écologique de sa mission, souhaite diminuer l'emballage et distribue ses produits de manière à valoriser le zéro déchet selon le principe de l'achat en vrac. Le consommateur doit alors apporter ses propres contenants réutilisables pour collecter ses aliments. Ce contexte d'achat a pour conséquence de complexifier le travail de ce consommateur, qui doit désormais prendre en charge des actions d'emballage traditionnellement attribuées à la distribution des produits. Le client doit ainsi apporter plusieurs contenants qu'il remplit et rapporte ensuite par lui-même à son domicile. Les périodes de commande étant peu fréquentes et les quantités achetées significatives, ce dernier doit sortir de sa zone de confort et faire preuve d'organisation dans la planification de ses commandes et d'initiatives afin de transporter ses emplettes, qui peuvent souvent peser plus de 20 livres. Lors de l'observation d'une journée d'emballage, il a été fréquent d'observer les clients transporter des sacs à dos de randonnée, des sacs à roulettes ou même des valises pour rapporter leurs vivres.

Au-delà de son rôle d'acheteur, le client est aussi encouragé à s'impliquer bénévolement dans l'organisation. Il peut réaliser cette implication en aidant à distribuer les aliments pendant les périodes de collecte ou en participant au nettoyage des lieux. En échange de ses bonnes actions, il reçoit des rabais sur ses futurs achats. Cette incitation au bénévolat est basée sur la volonté d'élargir le rôle des acteurs et d'encourager la participation citoyenne. Comme le mentionne un gestionnaire :

Chacun fait sa part. Quand tu viens en tant que bénévole, tu participes avec la communauté à faire avancer le projet. Le défi, c'est de faire en sorte que les gens aient envie de s'impliquer avec nous dans ce projet-là en venant faire du bénévolat et en vivant l'expérience. (Gestionnaire 2)
Ce répondant poursuit en expliquant que les bénévoles ont plusieurs raisons de s'impliquer : environnementales, économiques, sociales ou personnelles. Ultimement, l'engagement dans les activités bénévoles est un élément constitutif de la communauté NousRire qui diminue la séparation naturelle entre employés et clients :

Les bénévoles ne viennent pas tous pour la même raison. Certains viennent pour favoriser le biologique et le local. Quelqu'un m'a dit qu'il venait pour une réduction sur sa facture et en même temps que ça lui vidait la tête. Il est dans une ambiance agréable et vit l'instant présent. NousRire, ce n'est pas juste d'un côté les clients, de l'autre les membres d'équipe. Il y a les participants, les membres d'équipe et les bénévoles, et on est la communauté ensemble. » (Gestionnaire 2)

Cette vision est corroborée par les propos d'un bénévole :

Voir un autre environnement, la façon dont fonctionnent les gens et comment ils sont accueillants. À force de revenir, les gens te reconnaissent. Ils te font des câlins. Moi, ça me fait du bien de faire partie d'une communauté et d'un projet super intéressant, car je n’avais pas ça dans ma vie à moi. (Bénévole 1)

NousRire repousse davantage les limites de la conception du client en offrant à tous les bénévoles la possibilité de consulter ses données financières. Ainsi, les clients peuvent aisément avoir accès aux données financières, qui ne sont normalement réservées qu'à certains membres internes d'une entreprise. Comme l'indique un gestionnaire :

[Le bilan financier], on l'a partagé aux bénévoles. Pour moi, c'est de les inclure complètement dans la dynamique de notre communauté interne parce qu'ils font partie de la communauté interne. (Gestionnaire 2)

Ainsi, le statut de " client traditionnel », c'est-à-dire une personne qui a pour fonction de procéder à l'acquisition de biens ou de services chez un détaillant donné (Baines, Fill et Page, 2012), est profondément remis en question chez NousRire. Cette fonction est en fait transcendée grâce à une complexification de son rôle, qui se manifeste par 
une plus grande implication de sa part dans le fonctionnement, les procédés et la communauté interne de l'entreprise. Cette complexification du rôle se retrouve même dans l'appellation du concept «client». En fait, au sein du Lexique typiquement NousRire, le mot « participant » est utilisé à la place du terme «client» afin de souligner son rôle plus actif.

\subsection{Quête du potentiel humain par une transformation de la gouvernance et par le développement des rôles clés de gestion}

$\mathrm{Au}$ fil des ans, NousRire a connu une croissance importante qui a amené les gestionnaires à repenser en profondeur son modèle de gouvernance. Selon leurs dires, à un certain moment, le noyau de gestionnaires n'arrivait plus à répondre à la demande, ce qui a engendré un ralentissement des activités ainsi qu'une augmentation générale du niveau de stress chez les gestionnaires et les employés. Des tensions ont aussi émergé au sein de l'équipe et le style de gestion devenait de plus en plus hiérarchique et autoritaire, ce que les gestionnaires souhaitaient éviter. Ils ont alors décidé, avec l'accord de toute l'équipe, d'entamer la transition vers l'holacratie. Cette transition a été entamée en novembre 2018.

L'holacratie $^{8}$ est un système de gouvernance avec une structure composée de sous-systèmes (de cercles et de rôles) à la fois autonomes et dépendants de plus grands systèmes. Les rôles et cercles disposent d'une certaine autonomie et d'une autorité sur son fonctionnement, tout en n'étant pas complètement indépendants. Ils ont des responsabilités envers la plus large structure dans laquelle ils s'inscrivent et de laquelle ils dépendent (Robertson, 2015). Ce modèle de gestion redistribue l'autorité. Les employés sont encouragés à prendre des décisions relatives à leur rôle sans obtenir l'approbation des dirigeants et en communiquant seulement avec les personnes concernées par la décision. Ce mode de gouvernance s'accompagne de processus de rencontre très structuré permettant d'aborder les tensions de chacun et de travailler en équipe efficacement.

Les tensions jouent un rôle crucial : elles représentent des signes indiquant comment l'organisation peut continuer d'évoluer pour atteindre sa mission (Robertson, 2015). Ce style de gestion met de l'avant le potentiel créatif, la capacité en tant qu'humain à sentir les dissonances ou tensions du moment présent et à voir le potentiel de changements (Robertson, 2015). En holacratie, l'évolution (ou l'élévation) de l'organisation est une quête significative (Laloux, 2014). Les travailleurs ont un pouvoir décisionnel central et participent activement à l'évolution de l'organisation, ce qui leur permet davantage d'émancipation au travail (Robertson, 2015; Laloux, 2014).

L'holacratie offrait donc une plus grande implication des employés dans le fonctionnement de NousRire par la décentralisation du pouvoir décisionnel et permettait aussi à chacun un plus grand potentiel d'épanouissement. Cette vision est exprimée par un gestionnaire: «L'outil holacratique en tant que tel est monté de manière à ce que chacun puisse prendre son plein pouvoir, à enlever les dynamiques de pouvoir [malsaines]. » (Gestionnaire 1)

Ce modèle de gouvernance se positionne en rupture avec l'approche traditionnelle de la gestion des organisations basée sur le respect des hiérarchies et sur la chaîne de commandement. Les propos de ce gestionnaire reflètent cette idée :

L'holacratie, c'est assez différent. C'est une manière de fonctionner qui nous impose de réfléchir. On peut vraiment être autonome dans notre manière de fonctionner. Pour moi, c'est compliqué parce qu'on ne nous forme pas à ça. On nous forme à être un peu comme des moutons; à bien obéir. Il y a un responsable qui dit cela, alors tu écoutes. Si tu veux prendre des initiatives, il faut que tu en parles à ton superviseur, qui en parle à son superviseur, et cetera, et tu n'as pas de liberté d'action. L'holacratie, pour ça, c'est agréable. Dans mon rôle, demain, si je veux créer un comité pour discuter de l'implication bénévole, eh bien, je n'ai pas besoin de demander à quelqu'un pour le faire. Je ne suis pas obligé de le faire valider par un supérieur. (Gestionnaire 2)

L'adoption de l'holacratie force aussi de manière très spécifique à revoir la conceptualisation des réunions, qui deviennent des processus fondamentaux au fonctionnement de l'organisation. Elles sont organisées de manière à ce que les employés soulèvent librement les tensions relatives à leur 
rôle. L'identification des tensions engage alors à l'action. Selon un gestionnaire, cette manière de fonctionner nécessite une certaine adaptation de la part des employés :

Parfois, pour les gens, c'est plus facile de chialer sur quelque chose sans nécessairement prendre les moyens pour le changer. C'est plus facile de mettre la faute sur les coéquipiers ou les patrons et de ne pas prendre action finalement. Quand c'est le mode de fonctionnement d'une personne depuis des années [...]. Eh bien, ici, on arrive dans quelque chose de différent. Ça demande de changer de posture intérieure, de comportement, de réaction. Ça bouge beaucoup de choses. Les processus de réunion pour arriver à ça sont très carrés et ça demande un cheminement personnel. (Gestionnaire 1)

Suivant la mise en œuvre de l'holacratie, le Guide du vivre ensemble a été rédigé collectivement à l'hiver 2019. Les membres se sont inspirés du processus du world café 9 pour diriger les discussions entourant la rédaction de ce guide. L'objectif du document était d'établir les diverses règles organisationnelles. Il précise entre autres choses les conditions liées aux avantages sociaux, comme le nombre de semaines de vacances des employés. Un gestionnaire résume ainsi l'esprit général de ce guide :

L'idée, ce n'est pas de faire quelque chose de traditionnel. On n'est pas dans un mode « punition police ». La réalité, c'est qu'on a besoin d'un cadre pour fonctionner ensemble. Alors, définissons ce qu'est le cadre. Que ce cadre ne soit pas imposé de la direction. C'est pour ça qu'on a fait un world café : pour impliquer les gens. En tant que collectif, de quoi a-t-on besoin? C'est quelque chose qui pourra évoluer dans le temps. (Gestionnaire 1)

Le guide contient aussi des conséquences établies collectivement, appelées " contraventions drôles ", advenant que les règles ne soient pas respectées. Le même gestionnaire poursuit son idée au sujet de ces contraventions :

Si ça fait plusieurs fois qu'il y a un manquement sur quelque chose et qu'on s'est mis d'accord, eh bien, on pige une conséquence. Ça pourrait être toi la prochaine personne qui lave les toilettes ou qui fait une batch de muffins pour tout le monde. » (Gestionnaire 1)

Avec les restructurations engendrées par la mise en œuvre de l'holacratie, deux postes clés ont été développés. Tout d'abord, afin de consolider l'importance du potentiel humain au sein de l'organisation, NousRire a créé un poste de gestion dédié entièrement à cette fonction. Ce poste a pour objectif de favoriser «des êtres heureux au travail évoluant dans un environnement où ils peuvent être pleinement eux-mêmes $»^{10}$ et pour fonctions la coordination des tâches liées à la gestion du personnel et à la mise en œuvre de l'holacratie au sein de l'organisation. Ce poste contient un certain nombre de responsabilités liées entre autres à la rémunération, au recrutement, aux arrivées et aux départs des membres de l'équipe, au coaching des équipes et des individus, à la facilitation des rencontres en intelligence collective, à l'organisation des rassemblements et à l'accompagnement des membres d'équipe dans la pratique holacratique ${ }^{11}$.

L'implémentation de l'holacratie a aussi impliqué la formalisation d'un poste dédié au rayonnement. Sa raison d'être est de « rayonner pour toucher et inspirer à un monde meilleur et à être rieur/rieuse $»^{12}$. Les axes stratégiques associés à ce poste sont principalement liés à l'optimisation de la communication sur le Web, à l'affichage stratégique, aux kiosques dans des expos, à l'organisation d'événements et à l'activation du réseau de partenaires ${ }^{13}$.

Ainsi, suivant la logique du marketing traditionnel, le rayonnement vise à faire connaître NousRire. Par contre, ce qui est original chez NousRire et qui explique le mot « rayonnement» plutôt que « marketing» est la façon de développer la clientèle. NousRire veut, à travers sa mission écoresponsable et son mode de fonctionnement unique, inspirer les gens à s'impliquer avant tout. Elle se concentre sur son fonctionnement interne, sur sa mission et sur ses valeurs pour attirer les gens interpellés par ses actions, par son «amour». Un gestionnaire l'exprime en ces termes:

On dit qu'on rayonne. On est comme un soleil qui rayonne, au lieu d'être une carabine qui cible. La comparaison est bizarre... On est plus de l'intérieur vers l'extérieur, plutôt que de l'extérieur vers l'intérieur, d'aller chercher comme dans le marketing conventionnel. On est beaucoup plus magnétiques, dans le sens qu'on va 
rayonner et attirer les personnes qui sont inspirées par le rayonnement qu'on donne, plutôt qu'électrique, qu'aller chercher les gens qu'on veut. C'est plus sain. C'est plus représentatif de l'amour. Quand tu as un trop-plein d'amour, tu as envie d'en donner. Aller chercher de l'amour, ça ne marche pas. Tu peux essayer de charmer quelqu'un par toutes sortes de stratégies, mais ça ne tiendra pas longtemps et c'est moins honnête. » (Gestionnaire 3)

\section{Conclusion}

NousRire est un cas singulier d'une marque présentant une forte proposition de valeur axée sur la responsabilité sociale de l'entreprise. D'une part, cette valeur réside dans l'offre de produits respectant l'environnement et la santé des clients par le vrac, par la minimisation des déchets et par l'offre de produits bios. Ces pratiques représentent ce qui est couramment conçu dans la littérature comme l'accent sur l'environnement externe de la marque responsable. D'autre part, cette marque innove en élargissant sa proposition de valeur grâce à une concentration plus proche de son environnement interne. Elle y arrive en développant un ensemble de pratiques concrètes liées au développement du potentiel humain des acteurs gravitant autour de sa marque. Ces pratiques sont regroupées sous trois axes centraux : 1) le développement de pratiques créatives améliorant le bien-être et centrées sur la socialisation et l'attention, 2) la reconception de la notion même de client et 3) une transformation radicale du modèle de gestion.

De plus, NousRire s'éloigne de la conception classique de l'économie marchande telle qu'elle est présentée dans la littérature (voir entre autres Ertz, Hallegatte et Bousquet, 2019). Pour ce faire, l'entreprise force le client à sortir de sa zone de confort, dans laquelle il ne fait que se présenter en entreprise et acquérir ses biens. Le modèle NousRire amène plutôt le client à complexifier son rôle, en le forçant à s'organiser et à innover pour occuper un rôle élargi (emballer et transporter des quantités significatives de produits) traditionnellement associé à la logistique et à la distribution. Ces pratiques ont pour conséquence un certain effacement des frontières entre consommateur et producteur. Le client devient un bénévole, un participant actif de l'organisation, ce qui diminue sa distance symbolique avec les employés, pratique que nous appellerons internalisation du client.

Cette internalisation amène le client à développer une forte relation de cocréation de la valeur (Prahalad et Ramaswamy, 2004). Selon Zwick, Bonsu et Darmody (2008), le marketing du XXI ${ }^{e}$ siècle implique une prise de contrôle de la part des consommateurs. La cocréation, devenue tendance en affaires, veut ultimement utiliser les consommateurs comme une source de compétences en les mettant au travail. Cette situation s'inscrit dans une redéfinition de la consommation comme étant de plus en plus fluide, évolutive et complexe. En effet, les consommateurs prennent une part plus active dans les échanges marchands en devenant des recycleurs, des composteurs, des fournisseurs de biens et services, des réparateurs et des producteurs (comme résumé par Ertz, Bousquet et Hallegatte, 2019).

L’idéologie de cocréation montre un besoin, face à un système capitaliste complexifié, de reconfigurer le marketing comme une technologie d'exploitation des consommateurs à des fins d'accumulation de profits (Zwick et collab., 2008). On n'a qu'à penser aux restaurants-minute, qui délèguent le service et le nettoyage à leurs clients. NousRire n'est toutefois pas à l'abri de potentielles dérives. La ligne est mince entre la création d'une communauté de bénévoles engagés envers une cause commune et l'exploitation de la force de travail de personnes désireuses de s'impliquer. Or, à la différence d'entreprises comme McDonald's, NousRire est une organisation qui répond fondamentalement à des problèmes environnementaux et sociaux. De plus, elle ne pourrait fonctionner sans le travail de ses bénévoles-clients. Cette relation de cocréation est basée sur une logique s'apparentant davantage à la symbiose qu'à l'exploitation. Au contraire, en faisant travailler ses clients, McDonald's se désinvestit de certaines étapes sans valeur ajoutée pour les consommateurs et qui profitent à l'entreprise. NousRire mise plutôt sur un rapprochement qui renouvelle l'importance du client et qui crée une valeur significative pour ce dernier, ce qui renforce son attachement et son engagement envers la marque et son intégration à sa communauté (Muñiz et O'Guinn, 2001). Cette internalisation du client en bénévole peut être considérée comme une extension des pratiques de création de valeur d'une 
communauté de marque identifiées par Schau, Muñiz et Arnould (2009).

En parallèle, NousRire a choisi d'investir dans le potentiel de ses employés grâce à l'adoption de l'holacratie. La littérature semble avoir traité pour l'instant de l'holacratie de manière peu critique, exception faite d'une discussion sur les difficultés managériales liées à l'adoption du modèle (Bernstein, Brunch, Canner et Lee, 2016). Au-delà de cette dimension, un style de gouvernance donnant un pouvoir décisionnel accru aux employés peut risquer de trop en demander à ces derniers. En ce sens, il y a lieu de se demander si la gestion des ressources humaines chez NousRire s'apparente aux nouvelles méthodes de management prônées par les entreprises de la Silicon Valley, où les employés sont amenés à se donner corps et âme à leur employeur, qui offre en retour un environnement de travail stimulant incluant des services de bien-être ou de care comme le yoga, la méditation et la pleine conscience (English-Lueck et Lueck Avery, 2017). La pleine conscience est liée, entre autres, à une diminution du stress et à une augmentation de la qualité de l'attention (Kabat-Zinn, 2013; Goleman et Davidson, 2018). C'est ainsi un outil intéressant du point de vue du développement du potentiel humain. Cependant, cet outil n'est pas sans risque dans un contexte organisationnel. Il peut rendre les employés « dociles » et les encourager, comme le souligne Purser (2019), à performer davantage et plus longtemps dans un système néolibéral d'exploitation, sans participer à la transformation du système.

Certaines des pratiques adoptées par NousRire s'apparentent à cette notion de pleine conscience. Or, à la différence des entreprises de la Silicon Valley, NousRire est un milieu de travail qui n'est pas basé sur l'hypercompétitivité d'employés hyperqualifiés issus des meilleures universités (English-Lueck et Lueck Avery, 2017). C'est plutôt une entreprise à l'environnement agréable où les conditions de travail sont discutées ensemble, par principe holacratique. Cela dit, NousRire doit rester vigilante pour ne pas donner une charge de travail trop grande aux employés et bénévoles.

Au final, NousRire représente une marque allant au-delà de la marque responsable traditionnelle évoluant dans une vision classique des échanges marchands. Ce type de marque fait plutôt partie de la catégorie émergente des marques à fort capital social. Selon Champniss et Vilà (2011), ces marques valorisent la responsabilité sociale fondamentalement comme motivation intrinsèque, et non comme stratégie commerciale. Elles considèrent le consommateur avant tout comme un citoyen ou «citoyen consommant» (p. 138). Finalement, plutôt que de centrer leur proposition de valeur sur les classiques 4 $\mathrm{P}^{14}$, Champniss et Vilà (2011) proposent que ces marques développent leur valeur selon le principe des 5I, soit, l'interconnexion (valorisation des relations entre les parties prenantes gravitant autour de la marque), l'inclusion (ouverture vers tous, décloisonnement des profils et des segments), l'intérêt (valorisation à long terme du capital social), l'ignition (renforcement du rôle de la marque afin de nourrir le capital social) et l'imagination (maximisation du dialogue entre les acteurs et la créativité afin de valoriser le capital social).

Dans le futur, une analyse détaillée d'autres marques originales comme NousRire serait bénéfique afin de développer une compréhension plus approfondie de cette conceptualisation élargie de la marque responsable.

\section{NOTES}

1 https://nousrire.com/a-propos-2

2 Nous remercions l'équipe de NousRire pour son accueil, son ouverture d'esprit, sa gentillesse et sa participation à cette recherche.

3 NousRire est une entreprise au sens traditionnel du terme, mais elle est aussi considérée dans la perspective marketing comme une marque à part entière en raison du fait qu'elle est la résultante d'un nom, d'un logo, de symboles ainsi que d'une promesse de valeur distincte adressée à des parties prenantes variées comme les employés et les consommateurs (Balmer et Gray, 2003), au même titre que Google, Wal-Mart, etc. (Kotler et Armstrong, 2018).

4 Période durant laquelle les employés organisent la logistique des commandes en prévision des journées d'emballage.

5 Pour respecter la confidentialité des personnes, nous utiliserons délibérément le terme « gestionnaire » au masculin afin de confondre le genre des informateurs. 
6 Les données ont été collectées avant la pandémie de la COVID-19. Depuis, l'entreprise a dû adapter certaines de ses pratiques liées aux journées d'emballage (p. ex., imposer l'emploi de sacs compostables fournis par l'entreprise). Notre article portant sur les pratiques liées au potentiel humain, nous avons fait le choix de ne pas couvrir davantage ces modifications temporaires.

7 https://nousrire.com/a-propos-2

8 Hola réfère à la fois au grec holon (" tout qui fait partie d'un tout») et à holarchie (" connexion entre les holons ») (Robertson, 2015). Cratie vient du grec kratia («pouvoir, règle ») (Oxford Dictionary of English, 2005).

9 Stratégie de communication non traditionnelle utilisée dans les organisations pour faciliter la discussion en transition entre petits et grands groupes, sur différents sujets, dans une ambiance décontractée (Chang et Chen, 2015).

10 Selon la rubrique « Raison d'être » de la page web sur le potentiel humain suivante : https:/ fr.glassfrog.com/ organizations/13630/orgnav/roles/10756938/overview

11 Selon la rubrique « Redevabilités » de la page web sur le potentiel humain suivante : https:/ fr.glassfrog.com/ organizations/13630/orgnav/roles/10756938/overview

12 Selon la rubrique « Raison d'être » de la page web sur le rayonnement suivante : https://fr.glassfrog.com/ organizations/13630/orgnav/roles/10756937

13 Selon la rubrique « Stratégies » de la page web sur le rayonnement suivante : https://fr.glassfrog.com/organizations/13630/ orgnav/roles/10756937

14 Expression tirée des quatre actions fondamentales du marketing mixte, soit le prix, la promotion, le produit et la distribution (ou place en anglais) (Kotler et Armstrong, 2018).

\section{RÉFÉRENCES}

Austin, J. E. et Seitanidi, M. M. (2014). Creating value in nonprofit-business collaborations: New thinking and practice. San Francisco, CA: Jossey-Bass.

Baines, P., Fill, C. et Page, K. (2012). Le marketing : des fondements à la pratique contemporaine. Bruxelles : De Boeck.

Balmer, J. M. T. et Gray, E. R. (2003). Corporate brands: What are they? What of them? European Journal of Marketing, 37(7/8), 972-997. https://doi.org/10.1108/03090560310477627

Belz, F.-M. et Peattie, K. (2012). Sustainability marketing: A global perspective (2e éd.). West Sussex, R.-U. : John Wiley \& Sons.

Bernstein, E., Brunch, J., Canner, N. et Lee, M. (2016). Beyond the holacracy hype. Harvard Business Review, juillet-août, 1-13. Repéré à https://hbr.org/2016/07/beyond-the-holacracy-hype

Bryman, A. (2015). Social research methods (5e éd). Oxford, R.-U. : Oxford University Press.

Champniss, G. et Vilà, F. R. (2011). Brand valued: How socially valued brands bold the key to a sustainable future and business success. West Sussex, R.-U: John Wiley \& Sons.

Chang, W. et Chen, S. (2015). The impact of world café on entrepreneurial strategic planning capability. Journal of Business Research, 68(6), 1283-1290. https://doi.org/10.1016/j.jbusres.2014.11.020

Davis, D. M. et Hayes, J. A. (2011). What are the benefits of mindfulness? A practice review of psychotherapy-related research. Psychotherapy, 48(2), 198-208. https://doi.org/10.1037/a0022062

Eisenhardt, K. M. (1989). Building theories from case study research. The Academy of Management Review, 14(4), 532-550. https://doi.org/10.2307/258557

English-Lueck, J. A. et Lueck Avery, M. (2017). Intensifying work and chasing innovation: Incorporating care in Silicon Valley. Anthropology of Work Review, 38(1), 40-49.

Repéré à https://anthrosource.onlinelibrary.wiley.com/doi/abs/10.1111/awr.12111

Ertz, M., Bousquet, J. et Hallegatte, D. (2019). Introduction. Dans M. Ertz, D. Hallegatte et J. Bousquet (dir.), Les reconfigurations de l'échange marchand (p. 1-14). Québec: Presses de l'Université du Québec.

Ertz, M., Hallegatte, D. et Bousquet, J. (2019). Retour vers le futur de l'échange marchand : tour d'horizon, enjeux et perspectives. Organisations \& Territoires, 28(2), 99-113. https://doi.org/10.1522/revueot.v28n2.1058

Fuller, D. A. (1999). Sustainable marketing: Managerial-ecological issues. Thousand Oaks/Londres/New Delhi: SAGE. 
Goleman, D. et Davidson, R. J. (2018). Altered traits: Science reveals how meditation changes your mind, brain, and body. New York, NY: Penguin.

Iannuzzi, A. (2012). Greener products: The making and marketing of sustainable brands. Boca Raton, FL: CRC Press.

Kabat-Zinn, J. (2003). Mindfulness-based interventions in context: Past, present, and future. Clinical Psychology: Science and Practice, 10(2), 144-156. https://doi.org/10.1093/clipsy/bpg016

Kotler, P. et Armstrong, G. (2018). Principles of marketing (17e éd.). New York, NY: Pearson.

Kotler, P. et Lee, N. (2005). Corporate social responsibility: Doing the most good for your company and your cause. Hoboken, NJ: John Wiley \& Sons.

Laloux, F. (2014). Reinventing organizations: A guide to creating organizations inspired by the next stage of buman consciousness. Bruxelles: Nelson Parker.

Muñiz, Jr. A. et O’Guinn, T. (2001). Brand community. Journal of Consumer Research, 27(4), 412-432. https://doi.org/10.1086/319618

Murray, A., Skene, K. et Haynes, K. (2017). The circular economy: An interdisciplinary exploration of the concept and application in a global context. Journal of Business Ethics, 140, 369-380. https://doi.org/10.1007/s10551-015-2693-2

Soanes, C. et Stevenson, A. (2005). Oxford Dictionary of English. Oxford: Oxford University Press.

Porter, M. E. et Kramer, M. R. (2006). Strategy and society: The link between competitive advantage and corporate social responsibility. Harvard Business Review, 84(12), 78-92. Repéré à https://hbr.org/2006/12/strategy-and-society-the-linkbetween-competitive-advantage-and-corporate-social-responsibility

Prahalad, C. K. et Ramaswamy, V. (2004). The future of competition: Co-creating unique value with customers. Boston, MA: Harvard Business Review Press.

Pringle, H. et Thompson, M. (2001). Brand spirit: How cause-related marketing builds brands. West Sussex, R.-U.: John Wiley \& Sons.

Purser, R. E. (2019). McMindfulness: How mindfulness became the new capitalist spirituality. Londres, R.-U.: Repeater.

Robertson, M. (2014). Sustainability: Principles and practice. Londres/New York: Routledge.

Robertson, B. J. (2015). Holacracy: The new management system for a rapidly changing world. New York, NY: Henry Holt \& Co.

Samli, A. C. (1992). Social responsibility in marketing: A proactive and profitable marketing management strategy. Westport, CT: Quorum Books.

Schau, H. J., Muñiz, A. M. et Arnould, E. J. (2009). How brand community practices create value. Journal of Marketing, 73(5), 30-51. http://dx.doi.org/10.1509/jmkg.73.5.30

Schrier, M. (2012). Qualitative content analysis in practice. [S. 1.]: Thousand Oaks, Ca: SAGE Publications Ltd.

Stewart, H. J. (2011). An identity-based approach to the sustainable corporate brand. Corporate Communications: An International Journal, 16(2), 139-149. https://doi.org/10.1108/13563281111141660

Strauss, A. L. et Corbin, J. (1998). Basics of qualitative research: Techniques and procedures for developing grounded theory (4e éd.). Thousand Oaks, CA: SAGE Publications Ltd.

Yin, R. K. (2017). Case study research and applications: Design and methods (6e éd.). Thousand Oaks, CA: SAGE.

Zwick, D., Bonsu, S. K. et Darmody, A. (2008). Putting consumers to work: «Co-creation » and new marketing governmentality. Journal of Consumer Culture, 8(2), 163-196. https://doi.org/10.1177/1469540508090089 